\title{
ANALYSIS OF THE INEXACT UZAWA ALGORITHMS FOR NONLINEAR SADDLE-POINT PROBLEMS
}

\author{
JIAN-LEI LI ${ }^{\otimes 1}$, TING-ZHU HUANG ${ }^{1}$ and LIANG LI ${ }^{1}$
}

(Received 7 May, 2009; revised 29 May, 2010)

\begin{abstract}
Inexact Uzawa algorithms for solving nonlinear saddle-point problems are proposed. A simple sufficient condition for the convergence of the inexact Uzawa algorithms is obtained. Numerical experiments show that the inexact Uzawa algorithms are convergent.
\end{abstract}

2000 Mathematics subject classification: primary 65F10.

Keywords and phrases: convergence analysis, inexact Uzawa algorithm, iterative methods, nonlinear saddle-point problems, preconditioner.

\section{Introduction}

This paper provides convergence analysis of the inexact Uzawa methods for solving the nonlinear saddle-point system

$$
H(x, y)=\left[\begin{array}{c}
F(x)+B^{T} y-f \\
B x-C y-g
\end{array}\right]=0,
$$

where $B$ is an $m \times n$ matrix with full row rank $(m \leq n), B^{T}$ is the adjoint of the matrix $B, C$ is an $m \times m$ symmetric positive semi-definite matrix, $f$ is a vector in $\mathbb{R}^{n}, g$ is a vector in $\mathbb{R}^{m}$ and $F: \mathbb{R}^{n} \rightarrow \mathbb{R}^{n}$ is a nonlinear strongly monotone function differentiable everywhere. The nonlinear saddle-point system of the form (1.1) arises frequently in electromagnetic Maxwell equations [9, 12], partial differential equations [21] and nonlinear optimization [20,33], for example,

$$
\left\{\begin{array}{l}
\min _{x \in \mathbb{R}^{n}}\{J(x)-(f, x)\}, \\
\text { s.t. } B x-C y=g,
\end{array}\right.
$$

\footnotetext{
${ }^{1}$ School of Mathematical Sciences, University of Electronic, Science and Technology of China, Chengdu, Sichuan 611731, PR China; e-mail: hnmaths@163.com, tingzhuhuang@126.com, plum_liliang@uestc.edu.cn.

(C) Australian Mathematical Society 2010, Serial-fee code 1446-1811/2010 $\$ 16.00$
} 
where $J(x)$ is the function satisfying $\nabla J(x)=F(x)$. When $F(x)=A x$ with $A$ being an $n \times n$ symmetric positive-definite matrix, (1.1) becomes the well-known linear saddle-point problem

$$
\left(\begin{array}{cc}
A & B^{T} \\
B & -C
\end{array}\right)\left(\begin{array}{l}
x \\
y
\end{array}\right)=\left(\begin{array}{l}
f \\
g
\end{array}\right)
$$

There has been a growing interest in preconditioned iterative methods for solving the linear saddle-point problem (1.3), see [1, 4-7, 11, 16-19, 22, 23, 29, 30, 32, 34]. The Uzawa-type algorithms $[1-4,7,8,13,16-18,22,23,29]$ have been widely investigated and used in scientific computing, because of simplicity, efficiency and minimal computer memory requirements.

Elman and Golub [18] gave an inexact Uzawa method for solving linear saddlepoint problems (1.3) and obtained its convergence result. Their preconditioned inexact Uzawa algorithm is defined as follows:

$$
\begin{aligned}
& \text { Given an initial approximation } y_{0} \text { of } y \\
& \text { for } i=0 \text { until convergence, do } \\
& \text { Compute } x_{i+1} \text { such that } A x_{i+1}=f-B^{T} y_{i}+\delta_{i} \\
& \text { Compute } y_{i+1}=y_{i}+\alpha Q^{-1}\left(B x_{i+1}-C y_{i}-g\right)
\end{aligned}
$$

enddo

The vector $\delta_{i}$ is the residual of the approximate solution $x_{i+1}$ to the system $A x=f-B^{T} y_{i}, \alpha$ is a positive step size and $Q$ is an $m \times m$ symmetric positivedefinite matrix.

Chen [10] extended the method (1.4) to solve the nonlinear saddle-point problem (1.1):

$$
\begin{aligned}
& \text { Given an initial approximation } y_{0} \text { of } y \\
& \text { for } i=0 \text { until convergence, do } \\
& \text { Compute } x_{i+1} \text { such that } F\left(x_{i+1}\right)=f-B^{T} y_{i}+\delta_{i} \\
& \text { Compute } y_{i+1}=y_{i}+\alpha_{i} Q_{i}^{-1}\left(B x_{i+1}-C y_{i}-g\right)
\end{aligned}
$$

enddo

The vector $\delta_{i}$ is again the residual of the approximate solution $x_{i+1}$ to the system $F(x)=f-B^{T} y_{i}, \alpha_{i}$ is a positive step size and $Q_{i}$ is an $m \times m$ symmetric positivedefinite matrix. Chen [10] also provided a deep analysis of convergence in the standard $n^{2}$-norm. The method for $\alpha_{i}=0, Q_{i}=I$ was introduced by Ciarlet [14]. In fact, in this case, it is not easy to solve for $x_{i+1}$ in every iterative step for the nonlinear function $F(x)$.

There are other inexact Uzawa algorithms for solving the linear saddle-point problem (1.3), see [7, 23]. Hu and Zou [24] extended the inexact Uzawa algorithm of [23] to solve the nonlinear saddle-point problem (1.1), and also studied the convergence rate of their algorithm with respect to the energy norm. However, the algorithm of [24] should also solve a nonlinear system $F\left(x_{i+1}\right)=b$ when computing 
$x_{i+1}$ in every step. Moreover, the algorithm is only suitable for $C=0$. In this paper, motivated by the inexact algorithms of $[7,8]$, we propose inexact Uzawa algorithms to solve the nonlinear saddle-point problem (1.1) and give the convergence rate of these algorithms with respect to the energy norm. The methods discussed in this paper belong to the family of Uzawa-type methods.

The rest of this paper is organized as follows. In Section 2 we present our algorithms and in Section 3 we analyze their convergence. Finally, in Section 4 we present the results of some numerical experiments.

\section{Algorithms}

First, we recall some existing results from [10, 15, 24, 28], which will be used in the subsequent analysis. Here $\mathbb{R}^{n}$ denotes the usual $n$-dimensional Euclidean space. For any $n \times n$ positive-definite matrix $G$, the symmetric part $G_{s}$ of the matrix $G$ is defined by

$$
G_{s}=\frac{1}{2}\left(G+G^{T}\right),
$$

$\|x\|_{G_{s}}$ represents the $G$-induced norm, namely $\|x\|_{G_{s}}=\left(G_{s} x, x\right)^{\frac{1}{2}}$ for all $x \in \mathbb{R}^{n}$, and $I$ is the identity matrix with appropriate dimension.

For the nonlinear system (1.1), in this paper, we always assume that $F(x)$ is differentiable everywhere, Lipschitzian and strongly monotone with modulus $\mu$, that is,

$$
(F(\xi)-F(\eta), \xi-\eta) \geq \mu\|\xi-\eta\|^{2} \text { for all } \xi, \eta \in \mathbb{R}^{n} .
$$

Chen [10] pointed out that the strong monotonicity property of $F$ ensures that the sequences $\left\{x_{i}\right\}$ and $\left\{y_{i}\right\}$ are well defined, that is, for any $y_{i} \in \mathbb{R}^{m}$, there exists a unique $x_{i}$ such that $F\left(x_{i}\right)=f-B^{T} y_{i}$. Let $D_{F}$ be the set of points where $F$ is differentiable, and let $\nabla F(\xi)$ be the gradient of $F$ at $\xi \in D_{F}$. The generalized Jacobian of $F$ at $x$ in the sense of Clarke [15] is defined by

$$
\partial F(x)=\operatorname{co} \partial_{B} F(x),
$$

where co $\partial_{B} F(x)$ denotes the convex hull of the set

$$
\partial_{B} F(x)=\left\{\lim _{\xi \rightarrow x, \xi \in D_{F}} \nabla F(x)\right\} .
$$

It is well known that if $F$ is locally Lipschitzian, then the following generalized mean-value theorem [15] holds: for any $\xi, \eta \in \mathbb{R}^{n}$,

$$
F(\xi)-F(\eta) \in \operatorname{co} \partial F(\overline{\xi \eta})(\xi-\eta),
$$

where $\overline{\xi \eta}$ is the line segment between $\xi$ and $\eta$, and

$$
\operatorname{co} \partial F(\overline{\xi \eta})=\operatorname{co}\{V \in \partial F(\zeta), \zeta \in \overline{\xi \eta}\} .
$$

The strong monotonicity property (2.2) shows that all matrices in $\partial F(\eta)$ are positive definite for any $\eta \in \mathbb{R}^{n}[25,28]$, that is, the following inequality holds for any 
$V \in \partial F(\eta)$

$$
(V \xi, \xi) \geq \mu(\xi, \xi) \quad \text { for all } \xi \in \mathbb{R}^{n}
$$

Then, as in $[10,28]$, for any $\xi \in \mathbb{R}^{n}$, there exists a positive-definite matrix $Q_{A} \in$ $\partial_{B} F(\xi+\alpha)$ such that

$$
\lim _{\alpha \rightarrow 0} \frac{\left\|F(\xi+\alpha)-F(\xi)-Q_{A} \alpha\right\|}{\|\alpha\|}=0 .
$$

Note that all the above descriptions of the properties of the nonlinear mapping $F: \mathbb{R}^{n} \longrightarrow \mathbb{R}^{n}$ are in terms of the $l^{2}$-norm. Hu and Zou [24] pointed out that it is more accurate to interpret these properties in terms of the energy norm, that is, the norm induced by the generalized Jacobian $A$ of $F$ at $\xi_{i}$. We denote the generalized Jacobian of $F$ at $x$ as $Q$, where $\{x, y\}$ is the exact solution of system (1.1). By (2.4), $A$ is a positive-definite operator. Hence, $A_{s}$ is a symmetric positive-definite operator. Assume that $A_{s}$ satisfies

$$
(A v, w) \leq \alpha\left(A_{s} v, v\right)^{1 / 2}\left(A_{s} w, w\right)^{1 / 2} \text { for all } v, w \in \mathbb{R}^{n},
$$

for some number $\alpha$. Clearly, $\alpha \geq 1$. Moreover, since $A_{s}$ is symmetric positive-definite, such an $\alpha$ always exists.

From (2.4) and (2.5), Hu and Zou [24] gave the following equation under the norm $\|x\|_{G}=(G x, x)^{\frac{1}{2}}$, where $G$ is a positive-definite matrix. For any $\xi \in \mathbb{R}^{n}$, there is a positive-definite matrix $A \in \partial_{B} F(\xi+\alpha)$ such that

$$
\lim _{\alpha \rightarrow 0} \frac{\|F(\xi+\alpha)-F(\xi)-A \alpha\|_{Q^{-1}}}{\|\alpha\|_{Q}}=0 .
$$

In fact, it is difficult to determine the exact description of the matrix $Q$, but we know the exact description of $A$, and we give another more reasonable equation which is similar to Equation (2.7). Since $F$ is differentiable everywhere, Lipschitzian and strongly monotone, then for any $\xi \in \mathbb{R}^{n}$ there is a positive-definite matrix $A \in$ $\partial_{B} F(\xi+\alpha)$ such that

$$
\lim _{\alpha \rightarrow 0} \frac{\|F(\xi+\alpha)-F(\xi)-A \alpha\|_{\left(A^{-1}\right)_{s}}}{\|\alpha\|_{A_{s}}}=0 .
$$

REMARK 2.1. Nonlinear saddle-point problems (1.1) arise from certain convex optimization problems, and numerical solutions of certain nonlinear partial differential equations; refer, for example, to [10].

Now, we define our inexact Uzawa algorithms for solving (1.1). These algorithms are motivated by the Uzawa iteration [7, 8] for linear saddle-point systems.

Algorithm 1. For given $x_{0} \in \mathbb{R}^{n}$ and $y_{0} \in \mathbb{R}^{m}$, the sequence $\left\{\left(x_{i}, y_{i}\right)\right\}$ is defined for $i=1,2, \ldots$ by

$$
\left\{\begin{array}{l}
x_{i+1}=x_{i}+A^{-1}\left(f-\left(F\left(x_{i}\right)+B^{T} y_{i}\right)\right), \\
y_{i+1}=y_{i}+Q_{B}^{-1}\left(B x_{i+1}-C y_{i}-g\right),
\end{array}\right.
$$

where $A \in \partial_{B} F\left(x_{i}\right)$, and $Q_{B}$ is a symmetric and positive-definite operator. 
In Algorithm 1, the inner iteration should compute the inverse of the matrix $A$. If $A^{-1}$ is difficult to compute, the computation of $A^{-1}$ can be replaced by that of an approximation to $A^{-1}$ obtained by applying a nonlinear iterative process for inverting $A$. In Algorithm 2, the nonlinear Uzawa algorithm is proposed for nonlinear saddle-point problems (1.1).

Algorithm 2. For given $x_{0} \in \mathbb{R}^{n}$ and $y_{0} \in \mathbb{R}^{m}$, the sequence $\left\{\left(x_{i}, y_{i}\right)\right\}$ is defined for $i=1,2, \ldots$ by

$$
\left\{\begin{array}{l}
x_{i+1}=x_{i}+\phi\left(f-\left(F\left(x_{i}\right)+B^{T} y_{i}\right)\right), \\
y_{i+1}=y_{i}+Q_{B}^{-1}\left(B x_{i+1}-C y_{i}-g\right),
\end{array}\right.
$$

where $Q_{B}$ is a symmetric and positive-definite operator, and $\phi(v)$ is an approximation to the solution $\xi$ of $A \xi=v$ which satisfies

$$
\left\|\phi(v)-A^{-1} v\right\|_{A_{s}} \leq \delta\left\|A^{-1} v\right\|_{A_{s}} \equiv \delta\|v\|_{\left(A^{-1}\right)_{s}}
$$

for some $\delta \in(0,1)$. The assumption (2.11) is satisfied by the approximate inverse associated with the GMRES method [31] for a nonsymmetric matrix $A$, the approximate inverse associated with the preconditioned conjugate gradient (PCG) algorithm [26] and the approximate inverse defined by one sweep of a multigrid algorithm with conjugate gradient smoothing for the symmetric case, see [7, 27].

To analyse and describe the convergence of the above two algorithms, we need to introduce some parameters. First, by (2.8) we know that, for any $x_{i} \in \mathbb{R}^{n}$, there is a positive number $\omega \in(0,1)$ such that

$$
\left\|F\left(x_{i}+\alpha\right)-F\left(x_{i}\right)-A \alpha\right\|_{\left(A^{-1}\right)_{s}} \leq \omega\|\alpha\|_{A_{s}} .
$$

In addition, we assume that, for the symmetric positive-definite matrix $Q_{B}$ and any $y \in \mathbb{R}^{m}, A \in \mathbb{R}^{n \times n}$, there exists a number $\gamma \in(0,1)$ such that

$$
(1-\gamma)\left(Q_{B} y, y\right) \leq\left(\left(B\left(A_{s}\right)^{-1} B^{T}+C\right) y, y\right) \leq\left(Q_{B} y, y\right) .
$$

In the following section, we determine the convergence rates of Algorithms 1 and 2.

\section{Convergence analysis of the Uzawa algorithms}

First, we give some lemmas for later use.

LeMma 3.1 ([8]). Suppose that $A$ is an invertible linear operator with positivedefinite symmetric part $A_{s}$ that satisfies (2.6). Then, $\left(A^{-1}\right)_{s}$ is positive-definite and satisfies

$$
\left(\left(A^{-1}\right)_{s} w, w\right) \leq\left(\left(A_{s}\right)^{-1} w, w\right) \leq \alpha^{2}\left(\left(A^{-1}\right)_{s} w, w\right) \quad \text { for all } w \in \mathbb{R}^{n} .
$$

LEMMA 3.2. For any $v \in \mathbb{R}^{n}$, if $A$ is positive-definite and $Q_{B}$ is symmetric positivedefinite, and if (2.13) is satisfied, then we have the following inequality:

$$
\|B v\|_{Q_{B}^{-1}} \leq\|v\|_{A_{s}} .
$$


Proof. For any $v \in \mathbb{R}^{n}$ and $w \in \mathbb{R}^{m}$, by (2.13), we have

$$
\begin{aligned}
\|B v\|_{Q_{B}^{-1}}^{2} & \equiv\left(Q_{B}^{-1} B v, B v\right)=\sup _{w \in \mathbb{R}^{m}} \frac{\left(Q_{B}^{-1} B v, w\right)^{2}}{\left(Q_{B}^{-1} w, w\right)} \\
& =\sup _{w \in \mathbb{R}^{m}} \frac{(B v, w)^{2}}{\left(Q_{B} w, w\right)}=\sup _{w \in \mathbb{R}^{m}} \frac{\left(A_{s}^{\frac{1}{2}} v, A_{s}^{-\frac{1}{2}} B^{T} w\right)^{2}}{\left(Q_{B} w, w\right)} \\
& \leq \sup _{w \in \mathbb{R}^{m}} \frac{\left(A_{s} v, v\right)\left(B\left(A_{s}\right)^{-1} B^{T} w, w\right)}{\left(Q_{B} w, w\right)} \\
& \leq\left(A_{s} v, v\right) \equiv\|v\|_{A_{s}}^{2} .
\end{aligned}
$$

Lemma 3.3. Suppose that $A$ is positive-definite and its symmetric part $A_{s}$ satisfies (2.6). Let $Q_{B}$ be a symmetric and positive-definite operator satisfying (2.13). For $\theta=\left(2-\left(2(1-\gamma) / \alpha^{2}\right)\right)^{\frac{1}{2}}$,

$$
\left\|\left(I-Q_{B}^{-1}\left(B A^{-1} B^{T}+C\right)\right) v\right\|_{Q_{B}} \leq \theta\|v\|_{Q_{B}} .
$$

Moreover, if $0 \leq \gamma \leq \frac{1}{2}$, then $\theta \leq 1$.

Proof. Let $L=B A^{-1} B^{T}$. Then

$$
\begin{aligned}
& \left\|\left(I-Q_{B}^{-1}\left(B A^{-1} B^{T}+C\right)\right) v\right\|_{Q_{B}}^{2} \\
& \quad=\|v\|_{Q_{B}}^{2}-2((L+C) v, v)+\left((L+C) v, Q_{B}^{-1}(L+C) v\right) .
\end{aligned}
$$

By (2.13) and (3.1), we obtain

$$
\begin{aligned}
(1-\gamma)\|v\|_{Q_{B}}^{2} & \leq\left(\left(A_{S}\right)^{-1} B^{T} v, B^{T} v\right)+(C v, v) \\
& \leq \alpha^{2}(L v, v)+(C v, v) \leq \alpha^{2}((L+C) v, v) .
\end{aligned}
$$

Equation (3.1) gives

$$
\begin{aligned}
\left(A^{-1} v, w\right) & =\left(\left(A_{S}\right)^{\frac{1}{2}} A^{-1} v,\left(A_{S}\right)^{-\frac{1}{2}} w\right) \leq\left\|\left(A_{s}\right)^{\frac{1}{2}} A^{-1} v\right\|\left\|\left(A_{s}\right)^{-\frac{1}{2}} w\right\| \\
& =\left(A^{-1} v, v\right)^{\frac{1}{2}}\left(\left(A_{S}\right)^{-1} w, w\right)^{\frac{1}{2}} \\
& \leq\left(\left(A_{S}\right)^{-1} v, v\right)^{\frac{1}{2}}\left(\left(A_{S}\right)^{-1} w, w\right)^{\frac{1}{2}} .
\end{aligned}
$$

Using (3.6), we obtain

$$
(L v, w)=\left(A^{-1} B^{T} v, B^{T} w\right) \leq\left(B\left(A_{s}\right)^{-1} B^{T} v, v\right)^{\frac{1}{2}}\left(B\left(A_{s}\right)^{-1} B^{T} w, w\right)^{\frac{1}{2}} .
$$

By the Cauchy-Schwarz inequality,

$$
(C v, w) \leq(C v, v)^{\frac{1}{2}}(C w, w)^{\frac{1}{2}}
$$


By (2.13), (3.7) and (3.8), and using the Cauchy-Schwarz inequality again, we get

$$
\begin{aligned}
((L+C) v, w) & \leq\left(\left(B\left(A_{s}\right)^{-1} B^{T}+C\right) v, v\right)^{\frac{1}{2}}\left(\left(B\left(A_{s}\right)^{-1} B^{T}+C\right) w, w\right)^{\frac{1}{2}} \\
& \leq\|v\|_{Q_{B}}\|w\|_{Q_{B}} .
\end{aligned}
$$

Let $w=Q_{B}^{-1}(L+C) v$. By (3.9),

$$
\left((L+C) v, Q_{B}^{-1}(L+C) v\right) \leq\|v\|_{Q_{B}}^{2} .
$$

Equations (3.4), (3.5) and (3.10) show that

$$
\left\|\left(I-Q_{B}^{-1}\left(B A^{-1} B^{T}+C\right)\right) v\right\|_{Q_{B}}^{2} \leq \theta^{2}\|v\|_{Q_{B}}^{2},
$$

where $\theta^{2}=\left(2-\left(2(1-\gamma) / \alpha^{2}\right)\right)$. It is easy to verify that if $0 \leq \gamma \leq \frac{1}{2}$, then $\theta \leq 1$. The proof of the lemma is completed.

For the convergence of the inexact Uzawa algorithm, we have the following theorems.

THEOREM 3.4. Assume that Equations (2.6) and (2.13) hold and $F$ is differentiable everywhere. Let $\{(x, y)\}$ be the solution pair for (1.1) and let $\left\{\left(x_{i}, y_{i}\right)\right\}$ be defined by the Uzawa Algorithm 1 and define the residuals $e_{i}^{x}=x_{i}-x, e_{i}^{y}=y_{i}-y$. Let $\theta=\left(2-(2(1-\gamma)) / \alpha^{2}\right)^{\frac{1}{2}}$. Then $x_{i}$ and $y_{i}$ converge to $x$ and $y$, respectively, if

$$
0<\omega<\frac{1}{2}, \quad 0<\theta<\frac{1-2 \omega}{1-\omega} .
$$

In this case, the following inequality holds:

$$
\omega\left(A_{s} e_{i+1}^{x}, e_{i+1}^{x}\right)+\left(Q_{B} e_{i+1}^{y}, e_{i+1}^{y}\right) \leq \rho^{2}\left(\omega\left(A_{s} e_{i}^{x}, e_{i}^{x}\right)+\left(Q_{B} e_{i}^{y}, e_{i}^{y}\right)\right),
$$

where

$$
\rho=\frac{\omega+\theta+\sqrt{(\omega+\theta)^{2}-4 \omega(\theta-1)}}{2} .
$$

PROOF. From Algorithm 1 and (1.1), we have the following equations:

$$
\begin{gathered}
e_{i+1}^{x}=e_{i}^{x}+A^{-1}\left(F(x)-F\left(x_{i}\right)-B^{T} e_{i}^{y}\right), \\
e_{i+1}^{y}=e_{i}^{y}+Q_{B}^{-1}\left(B e_{i+1}^{x}-C e_{i}^{y}\right) .
\end{gathered}
$$

Equation (3.14) gives

$$
e_{i+1}^{x}=A^{-1}\left(F(x)-F\left(x_{i}\right)+A e_{i}^{x}\right)-A^{-1} B^{T} e_{i}^{y} .
$$

Substituting for $e_{i+1}^{x}$ in (3.15) using the above equation,

$$
\begin{aligned}
e_{i+1}^{y} & =e_{i}^{y}+Q_{B}^{-1}\left[B A^{-1}\left(F(x)-F\left(x_{i}\right)+A e_{i}^{x}\right)-\left(B A^{-1} B^{T}+C\right) e_{i}^{y}\right] \\
& =\left[I-Q_{B}^{-1}\left(B A^{-1} B^{T}+C\right)\right] e_{i}^{y}+Q_{B}^{-1} B A^{-1}\left(F(x)-F\left(x_{i}\right)+A e_{i}^{x}\right) .
\end{aligned}
$$


In addition, by (2.12), we conclude that

$$
\left\|F(x)-F\left(x_{i}\right)+A e_{i}^{x}\right\|_{\left(A^{-1}\right)_{s}} \leq \omega\left\|e_{i}^{x}\right\|_{A_{s}} .
$$

It follows from the triangular inequality, (2.13), (3.1) and (3.17) that

$$
\begin{aligned}
\left\|e_{i+1}^{x}\right\|_{A_{s}} & \leq\left\|F(x)-F\left(x_{i}\right)+A e_{i}^{x}\right\|_{\left(A^{-1}\right)_{s}}+\left\|A^{-1} B^{T} e_{i}^{y}\right\|_{A_{s}} \\
& \leq \omega\left\|e_{i}^{x}\right\|_{A_{s}}+\left\|B^{T} e_{i}^{y}\right\|_{\left(A^{-1}\right)_{s}} \\
& \leq \omega\left\|e_{i}^{x}\right\|_{A_{s}}+\left\|e_{i}^{y}\right\|_{Q_{B}} .
\end{aligned}
$$

Using the triangular inequality, from (3.2), (3.3), (3.16) and (3.17),

$$
\begin{aligned}
\left\|e_{i+1}^{y}\right\|_{Q_{B}} & \leq\left\|\left(I-Q_{B}^{-1}\left(B A^{-1} B^{T}+C\right)\right) e_{i}^{y}\right\|_{Q_{B}}+\left\|A^{-1}\left(F(x)-F\left(x_{i}\right)+A e_{i}^{x}\right)\right\|_{A_{s}} \\
& \leq \omega\left\|e_{i}^{x}\right\|_{A_{s}}+\theta\left\|e_{i}^{y}\right\|_{Q_{B}} .
\end{aligned}
$$

It follows from (3.18) and (3.19) that

$$
\left(\begin{array}{c}
\left\|e_{i+1}^{x}\right\|_{A_{s}} \\
\left\|e_{i+1}^{y}\right\| Q_{B}
\end{array}\right) \leq M\left(\begin{array}{c}
\left\|e_{i}^{x}\right\|_{A_{s}} \\
\left\|e_{i}^{y}\right\|_{Q_{B}}
\end{array}\right),
$$

where $M$ is given by

$$
M=\left(\begin{array}{ll}
\omega & 1 \\
\omega & \theta
\end{array}\right) .
$$

Obviously, $M$ is symmetric with respect to the inner product [., .] on $\mathbb{R}^{2}$ defined by

$$
\begin{aligned}
{\left[\left(\begin{array}{l}
x_{1} \\
y_{1}
\end{array}\right),\left(\begin{array}{l}
x_{2} \\
y_{2}
\end{array}\right)\right] } & \equiv\left(\left(\begin{array}{ll}
\omega & 0 \\
0 & 1
\end{array}\right)\left(\begin{array}{l}
x_{1} \\
y_{1}
\end{array}\right),\left(\begin{array}{l}
x_{2} \\
y_{2}
\end{array}\right)\right) \\
& =\omega x_{1} x_{2}+y_{1} y_{2} .
\end{aligned}
$$

Thus, from (3.20),

$$
\begin{aligned}
& \omega\left(A_{s} e_{i+1}^{x}, e_{i+1}^{x}\right)+\left(Q_{B} e_{i+1}^{y}, e_{i+1}^{y}\right)=\left[\left(\begin{array}{c}
\left\|e_{i+1}^{x}\right\|_{A_{s}} \\
\left\|e_{i+1}^{y}\right\|_{Q_{B}}
\end{array}\right),\left(\begin{array}{c}
\left\|e_{i+1}^{x}\right\|_{A_{s}} \\
\left\|e_{i+1}^{y}\right\|_{Q_{B}}
\end{array}\right)\right] \\
& \leq\left.M\left(\begin{array}{c}
\left\|e_{i}^{x}\right\|_{A_{s}} \\
\left\|e_{i}^{y}\right\|_{Q_{B}}
\end{array}\right), M\left(\begin{array}{c}
\left\|e_{i}^{x}\right\|_{A_{s}} \\
\left\|e_{i}^{y}\right\|_{Q_{B}}
\end{array}\right)\right] \\
& \leq \rho^{2}\left(\omega\left(A_{s} e_{i}^{x}, e_{i}^{x}\right)+\left(Q_{B} e_{i}^{y}, e_{i}^{y}\right)\right),
\end{aligned}
$$

where $\rho$ is the spectral radius of $M$. The eigenvalues of $M$ are the roots of

$$
\lambda^{2}-(\omega+\theta) \lambda+\omega(\theta-1)=0
$$

From the above equation, we know that $\lambda \in \mathbb{R}$ and $\omega+\theta>0$. Obviously, the spectral radius $\rho$ of $M$ is equal to its positive eigenvalue which is given by (3.13).

It is easy to see that if (3.11) is satisfied, then $\rho<1$. This completes the proof of the theorem. 
THEOREM 3.5. Assume that $F$ is differentiable everywhere, A is the Jacobian matrix of $F$ at $x_{i}$ and satisfies (2.6), and inequalities (2.11), (2.12) and (2.13) hold. Let $\{(x, y)\}$ be the solution pair for (1.1) and let $\left\{\left(x_{i}, y_{i}\right)\right\}$ be defined by the nonlinear Uzawa Algorithm 2 and with residuals denoted by $e_{i}^{x}=x_{i}-x, e_{i}^{y}=y_{i}-y$. Let $\theta=\left(2-\left(2(1-\gamma) / \alpha^{2}\right)\right)^{\frac{1}{2}}$. Then $x_{i}$ and $y_{i}$ converge to $x$ and $y$, respectively, if

$$
0<\omega<\frac{1}{2}, \quad 0<\theta<\frac{1-2 \omega}{1-\omega}, \quad 0<\delta<\frac{1-\theta+\omega(\theta-2)}{3-\theta+\omega(2-\theta)} .
$$

In this case, the following inequality holds:

$$
\begin{aligned}
(\omega+ & \delta+\omega \delta)\left(A_{s} e_{i+1}^{x}, e_{i+1}^{x}\right)+(\delta+1)\left(Q_{B} e_{i+1}^{y}, e_{i+1}^{y}\right) \\
& \leq \rho^{2}\left((\omega+\delta+\omega \delta)\left(A_{s} e_{i}^{x}, e_{i}^{x}\right)+(\delta+1)\left(Q_{B} e_{i}^{y}, e_{i}^{y}\right)\right)
\end{aligned}
$$

where

$$
\rho=\frac{2 \delta+\theta+\omega+\omega \delta+\sqrt{(2 \delta+\theta+\omega+\omega \delta)^{2}-4(\omega+\delta+\omega \delta)(\theta-1)}}{2} .
$$

PROOF. By (2.12), we conclude that

$$
\left\|F(x)-F\left(x_{i}\right)+A e_{i}^{x}\right\|_{\left(A^{-1}\right)_{s}} \leq \omega\left\|e_{i}^{x}\right\|_{A_{s}} .
$$

From Algorithm 2 and (1.1), we have the following equations:

$$
\begin{gathered}
e_{i+1}^{x}=e_{i}^{x}+\phi\left(F(x)-F\left(x_{i}\right)-B^{T} e_{i}^{y}\right), \\
e_{i+1}^{y}=e_{i}^{y}+Q_{B}^{-1}\left(B e_{i+1}^{x}-C e_{i}^{y}\right) .
\end{gathered}
$$

Equation (3.25) gives

$$
e_{i+1}^{x}=\left(\phi-A^{-1}\right)\left(F(x)-F\left(x_{i}\right)-B^{T} e_{i}^{y}\right)+A^{-1}\left(F(x)-F\left(x_{i}\right)+A e_{i}^{x}-B^{T} e_{i}^{y}\right) .
$$

Substituting for $e_{i+1}^{x}$ in (3.26) using the above equation,

$$
\begin{aligned}
e_{i+1}^{y}= & e_{i}^{y}+Q_{B}^{-1}\left(B e_{i+1}^{x}-C e_{i}^{y}\right) \\
= & {\left[I-Q_{B}^{-1}\left(B A^{-1} B^{T}+C\right)\right] e_{i}^{y}+Q_{B}^{-1} B\left[\left(\phi-A^{-1}\right)\left(F(x)-F\left(x_{i}\right)-B^{T} e_{i}^{y}\right)\right.} \\
& \left.\quad+A^{-1}\left(F(x)-F\left(x_{i}\right)+A e_{i}^{x}\right)\right] .
\end{aligned}
$$

It follows from the triangular inequality, (2.13) and (3.24) that we have

$$
\begin{aligned}
\left\|e_{i+1}^{x}\right\|_{A_{s} \leq} \leq & \left\|\left(\phi-A^{-1}\right)\left(F(x)-F\left(x_{i}\right)-B^{T} e_{i}^{y}\right)\right\|_{A_{s}} \\
& +\left\|F(x)-F\left(x_{i}\right)+A e_{i}^{x}-B^{T} e_{i}^{y}\right\|_{\left(A^{-1}\right)_{s}} \\
\leq & \delta\left(\left\|F(x)-F\left(x_{i}\right)+A e_{i}^{x}\right\|_{\left(A^{-1}\right)_{s}}+\left\|A e_{i}^{x}+B^{T} e_{i}^{y}\right\|_{\left(A^{-1}\right)_{s}}\right) \\
& \quad+\left\|F(x)-F\left(x_{i}\right)+A e_{i}^{x}\right\|_{\left(A^{-1}\right)_{s}}+\left\|e_{i}^{y}\right\|_{Q_{B}} \\
\leq & \delta \omega\left\|e_{i}^{x}\right\|_{A_{s}}+\delta\left(\left\|e_{i}^{x}\right\|_{A_{s}}+\left\|e_{i}^{y}\right\|_{Q_{B}}\right)+\omega\left\|e_{i}^{x}\right\|_{A_{s}}+\left\|e_{i}^{y}\right\|_{Q_{B}} \\
= & (\omega+\delta+\omega \delta)\left\|e_{i}^{x}\right\|_{A_{s}}+(\delta+1)\left\|e_{i}^{y}\right\|_{Q_{B}} .
\end{aligned}
$$


Using the triangular inequality, from (2.13), (3.27), Lemmas 3.2 and 3.3,

$$
\begin{aligned}
\left\|e_{i+1}^{y}\right\|_{Q_{B}} \leq & \theta\left\|e_{i}^{y}\right\|_{Q_{B}}+\delta\left\|F(x)-F\left(x_{i}\right)-B^{T} e_{i}^{y}\right\|_{\left(A^{-1}\right)_{s}} \\
& +\left\|F(x)-F\left(x_{i}\right)+A e_{i}^{x}\right\|_{\left(A^{-1}\right)_{s}} \\
\leq & \omega\left\|e_{i}^{x}\right\|_{A_{s}}+\theta\left\|e_{i}^{y}\right\|_{Q_{B}}+\delta\left(\omega\left\|e_{i}^{x}\right\|_{A_{s}}+\left\|e_{i}^{x}\right\|_{A_{s}}+\left\|e_{i}^{y}\right\|_{Q_{B}}\right) \\
= & (\omega+\delta+\omega \delta)\left\|e_{i}^{x}\right\|_{A_{s}}+(\delta+\theta)\left\|e_{i}^{y}\right\|_{Q_{B}} .
\end{aligned}
$$

It follows from (3.28) and (3.29) that

$$
\left(\begin{array}{l}
\left\|e_{i+1}^{x}\right\|_{A_{s}} \\
\left\|e_{i+1}^{y}\right\|_{Q_{B}}
\end{array}\right) \leq M\left(\begin{array}{c}
\left\|e_{i}^{x}\right\|_{A_{s}} \\
\left\|e_{i}^{y}\right\|_{Q_{B}}
\end{array}\right),
$$

where $M$ is given by

$$
M=\left(\begin{array}{ll}
\omega+\delta+\omega \delta & \delta+1 \\
\omega+\delta+\omega \delta & \delta+\theta
\end{array}\right)
$$

Obviously, $M$ is symmetric with respect to the inner product [., .] on $\mathbb{R}^{2}$ defined by

$$
\begin{aligned}
{\left[\left(\begin{array}{l}
x_{1} \\
y_{1}
\end{array}\right),\left(\begin{array}{l}
x_{2} \\
y_{2}
\end{array}\right)\right] } & \equiv\left(\left(\begin{array}{cc}
\omega+\delta+\omega \delta & 0 \\
0 & \delta+1
\end{array}\right)\left(\begin{array}{l}
x_{1} \\
y_{1}
\end{array}\right),\left(\begin{array}{l}
x_{2} \\
y_{2}
\end{array}\right)\right) \\
& =(\omega+\delta+\omega \delta) x_{1} x_{2}+(\delta+1) y_{1} y_{2} .
\end{aligned}
$$

Thus, from (3.30),

$$
\begin{aligned}
(\omega+ & \delta+\omega \delta)\left(A_{s} e_{i+1}^{x}, e_{i+1}^{x}\right)+(\delta+1)\left(Q_{B} e_{i+1}^{y}, e_{i+1}^{y}\right) \\
= & {\left[\left(\begin{array}{c}
\left\|e_{i+1}^{x}\right\|_{A_{s}} \\
\left\|e_{i+1}^{y}\right\|_{Q_{B}}
\end{array}\right),\left(\begin{array}{c}
\left\|e_{i+1}^{x}\right\|_{A_{s}} \\
\left\|e_{i+1}^{y}\right\|_{Q_{B}}
\end{array}\right)\right] } \\
\leq & \left.M\left(\begin{array}{c}
\left\|e_{i}^{x}\right\|_{A_{s}} \\
\left\|e_{i}^{y}\right\|_{Q_{B}}
\end{array}\right), M\left(\begin{array}{c}
\left\|e_{i}^{x}\right\|_{A_{s}} \\
\left\|e_{i}^{y}\right\|_{Q_{B}}
\end{array}\right)\right] \\
& \leq \rho^{2}\left((\omega+\delta+\omega \delta)\left(A_{s} e_{i}^{x}, e_{i}^{x}\right)+(\delta+1)\left(Q_{B} e_{i}^{y}, e_{i}^{y}\right)\right),
\end{aligned}
$$

where $\rho$ is the spectral radius of $M$. The eigenvalues of $M$ are the roots of

$$
\lambda^{2}-(2 \delta+\theta+\omega+\omega \delta) \lambda+(\omega+\delta+\omega \delta)(\theta-1)=0
$$

From the above equation, we know that $\lambda \in \mathbb{R}$ and $2 \delta+\gamma+\omega+\omega \delta>0$. Obviously, the spectral radius $\rho$ of $M$ is equal to its positive eigenvalue which is given by (3.23).

It is easy to see that if (3.21) is satisfied, then $\rho<1$. This completes the proof of the theorem.

REMARK 3.6. In Theorems 3.4 and 3.5, the initial guess $\left\{\left(x_{0}, y_{0}\right)\right\}$ is required to lie within a small neighbourhood of the exact solution $\{(x, y)\}$. 


\section{Numerical experiments}

In this section, we consider the numerical example of the nonlinear saddle-point problem (1.1) described in [24] to illustrate the convergence of Algorithms 1 and 2. For completeness, we describe the example again.

Let $I_{m}$ be the $m \times m$ identity matrix and let $T_{m}$ be an $m \times m$ matrix with entries given by

$$
t_{i j}= \begin{cases}1 & \text { if }|i-j|=1 \\ 0 & \text { otherwise }\end{cases}
$$

For $n=2 m$, we define an $n \times n$ symmetric positive-definite matrix $E$, an $m \times n$ matrix $B$ with full rank and an $m \times m$ symmetric positive semi-definite matrix $C$ as follows:

$$
E=\left(\begin{array}{cc}
\frac{5}{2} I_{m}-\frac{1}{4} T_{m} & -I_{m} \\
-I_{m} & \frac{5}{2} I_{m}-\frac{1}{4} T_{m}
\end{array}\right), \quad B=\left(0,2 I_{m}-T_{m}\right), \quad C=\left(\begin{array}{cc}
I_{m} / 2 & 0 \\
0 & 0
\end{array}\right) .
$$

The smallest and largest eigenvalues of $E$ are [24]

$$
\begin{aligned}
& \lambda_{1}=4 \sin ^{2} \frac{m \pi}{2(n+m)}+\sin ^{2} \frac{\pi}{2(1+m)}=1+\sin ^{2} \frac{\pi}{2(1+m)}, \\
& \lambda_{n}=4 \sin ^{2} \frac{n \pi}{2(n+m)}+\sin ^{2} \frac{m \pi}{2(1+m)}=3+\sin ^{2} \frac{m \pi}{2(1+m)} .
\end{aligned}
$$

The nonlinear mapping $F$ is defined as

$$
\begin{gathered}
F(x)=E x+\frac{1}{5}\left(\frac{x_{1}}{1+x_{1}^{2}}, \frac{x_{2}}{1+x_{2}^{2}}, \ldots, \frac{x_{n}}{1+x_{n}^{2}}\right)^{T}, \\
\text { for all } x=\left(x_{1}, x_{2}, \ldots, x_{n}\right)^{T} \in \mathbb{R}^{n} .
\end{gathered}
$$

It is easy to verify that $F$ is strongly monotone and Lipschitz continuous, see [24]. For any given $f \in \mathbb{R}^{n}$ and $g \in \mathbb{R}^{m}$, the system (1.1) has a unique solution. Moreover,

$$
A=E+\frac{1}{5} \operatorname{diag}\left(\frac{1-x_{1}^{2}}{\left(1+x_{1}^{2}\right)^{2}}, \frac{1-x_{2}^{2}}{\left(1+x_{2}^{2}\right)^{2}}, \ldots, \frac{1-x_{n}^{2}}{\left(1+x_{n}^{2}\right)^{2}}\right)
$$

which implies

$$
\frac{4}{5}\|\eta\|^{2} \leq(A \eta, \eta) \leq \frac{21}{5}\|\eta\|^{2}
$$

The functions $f$ and $g$ in (1.1) are generated using (1.1) when the exact solution is taken to be

$$
x=(1,1, \ldots, 1)^{T}, \quad y=\left(1, \frac{1}{2}, \ldots, \frac{1}{m}\right)^{T} .
$$

In our numerical experiments, the size of the problem is determined by the dimension $m$. We choose the zero vector as the initial guess vector $\left(x_{0}, y_{0}\right)$. The iterations of Algorithms 1 and 2 terminate when

$$
\text { Error }=\left\{\frac{\left\|f-F\left(x_{i}\right)-B^{T} y_{i}\right\|^{2}+\left\|g-B x_{i}+C y_{i}\right\|^{2}}{\|f\|^{2}+\|g\|^{2}}\right\}^{\frac{1}{2}} \leq 10^{-6} .
$$


TABLE 1. Iteration number and CPU time for IUA and NUA.

\begin{tabular}{rcc}
\hline \multicolumn{1}{c}{$m$} & IUA & NUA \\
\hline 50 & $28(0.0470)$ & $28(0.0780)$ \\
100 & $28(0.0940)$ & $28(0.1090)$ \\
200 & $27(0.2030)$ & $27(0.1710)$ \\
400 & $26(0.5000)$ & $26(0.2820)$ \\
500 & $26(0.6880)$ & $26(0.3440)$ \\
800 & $25(1.3590)$ & $25(0.5000)$ \\
1000 & $25(1.9530)$ & $25(0.5930)$ \\
2000 & $24(6.2820)$ & $24(1.1250)$ \\
4000 & $23(22.4220)$ & $23(2.0790)$ \\
5000 & $23(38.6410)$ & $23(2.7030)$ \\
8000 & $22(99.1410)$ & $23(4.9220)$ \\
9000 & $22(102.2190)$ & $22(5.3120)$ \\
\hline
\end{tabular}

In both algorithms, we choose the preconditioner $Q_{B}=\frac{5}{4} B B^{T}+C$, which ensures that inequality (2.13) holds. IUA denotes the inexact Uzawa Algorithm 1. NUA denotes the nonlinear Uzawa Algorithm 2 only using the nonlinear approximation to $A^{-1}$. In NUA, $\phi$ is defined by five steps of the PCG with preconditioner $M=L L^{T}$ applied to approximate the action of $A^{-1}$, where $L$ is the incomplete Cholesky factor of $E$, that is, $E=L L^{T}-R$, with drop tolerance 0.01 .

Numerical results are obtained using Matlab 7.0 on a personal computer with an Intel(R) Pentium(R) D 3.00 GHz CPU and 1 GB memory. We restrict our attention to the convergence of the IUA and NUA for nonlinear saddle-point problems. In Table 1 we report the number of iterations and the total computational time in seconds. Our numerical experiments illustrate the convergence theory developed in Section 3. From Table 1, we see that the number of iterations is virtually constant, the CPU time increases as $m$ increases for both algorithms, and the NUA is better than the IUA. In fact, the preconditioner $Q_{B}$ of the approximate Schur complement $B\left(A_{s}\right)^{-1} B^{T}+C$ plays an important role in the two algorithms, but, in practice, $Q_{B}$ is difficult to choose. Hence, we will attempt to find other iterative methods for various nonlinear saddlepoint problems in further research.

\section{Acknowledgements}

The authors thank the referee very much for comments and constructive suggestions, which were valuable for improving the quality of our manuscript. This research was supported by NSFC $(10926190,60973015)$ and the Sichuan Province Science and Technology Research Project (2009GZ0004, 2009HH0025).

\section{References}

[1] K. Arrow, L. Hurwicz and H. Uzawa, Studies in linear and nonlinear programming (Stanford University Press, Stanford, CA, 1958). 
[2] C. Bacuta, "A unified approach for Uzawa algorithms", SIAM J. Numer. Anal. 44 (2006) 2633-2649.

[3] C. Bacuta, "Schur complements on Hilbert spaces and saddle point systems", J. Comput. Appl. Math. 225 (2009) 581-593.

[4] Z. Z. Bai, B. N. Parlett and Z. Q. Wang, "On generalized successive overrelaxation methods for augmented linear systems", Numer. Math. 102 (2005) 1-38.

[5] M. Benzi, G. H. Golub and J. Liesen, "Numerical solution of saddle point problems", Acta Numer. 14 (2005) 1-137.

[6] J. H. Bramble and J. E. Pasciak, "A preconditioning technique for indefinite systems resulting from mixed approximations of elliptic problems", Math. Comp. 50 (1988) 1-17.

[7] J. H. Bramble, J. E. Pasciak and A. T. Vassilev, "Analysis of the inexact Uzawa algorithm for saddle point problems", SIAM J. Numer. Anal. 34 (1997) 1072-1092.

[8] J. H. Bramble, J. E. Pasciak and A. T. Vassilev, "Uzawa type algorithms for nonsymmetric saddle point problems", Math. Comp. 69 (1999) 667-689.

[9] K. H. Chan, K. Zhang, J. Zou and G. Schubert, "A non-linear, 3-D spherical $\alpha^{2}$ dynamo using a finite element method", Phys. Earth Planetary Int. 128 (2001) 35-50.

[10] X. J. Chen, "Global and superlinear convergence of inexact Uzawa methods for saddle point problems with nondifferentiable mappings", SIAM J. Numer. Anal. 35 (1998) 1130-1148.

[11] X. J. Chen, "On preconditioned Uzawa methods and SOR methods for saddle point problems", J. Comput. Appl. Math. 100 (1998) 207-224.

[12] Z. Chen, Q. Du and J. Zou, "Finite element methods with matching and nonmatching meshes for Maxwell equations with discontinuous coefficients", SIAM J. Numer. Anal. 37 (2000) $1542-1570$.

[13] X. L. Cheng, "On the nonlinear inexact Uzawa algorithm for saddle-Point problems", SIAM J. Numer. Anal. 37 (2000) 1930-1934.

[14] P. G. Ciarlet, Introduction to numerical linear algebra and optimization (Cambridge University Press, Cambridge, 1989).

[15] F. H. Clarke, Optimization and nonsmooth analysis (John Wiley, New York, 1983).

[16] M. R. Cui, "A sufficient condition for the inexact Uzawa algorithm for saddle point problems", J. Comput. Appl. Math. 139 (2002) 189-196.

[17] M. R. Cui, "Analysis of iterative algorithms of Uzawa type for saddle point problems", Appl. Numer. Math. 50 (2004) 133-146.

[18] H. C. Elman and G. H. Golub, "Inexact and preconditioned Uzawa algorithms for saddle point problems", SIAM J. Numer. Anal. 31 (1994) 1645-1661.

[19] H. C. Elman and D. J. Silvester, "Fast nonsymmetric iterations and preconditioning for NavierStoke equations", SIAM J. Sci. Comput. 17 (1996) 33-46.

[20] R. Fletcher, Practical methods of optimization, 2nd edn (John Wiley, Chichester, 1987).

[21] V. Girault and P. A. Raviart, Finite element methods for Navier-Stokes equations (Springer, Berlin, Heidelberg, 1986).

[22] G. H. Golub, X. Wu and J. Y. Yuan, "SOR-like methods for augmented systems", BIT. Numer. Math. 41 (2001) 71-85.

[23] Q. Y. Hu and J. Zou, "Two new variants of nonlinear inexact Uzawa algorithms for saddle point problems", Numer. Math. 93 (2002) 333-359.

[24] Q. Y. Hu and J. Zou, "Nonlinear inexact Uzawa algorithms for linear and nonlinear saddle point problems", SIAM J. Optim. 16 (2006) 798-825.

[25] H. Jiang and L. Qi, "Local uniqueness and Newton-type methods for nonsmooth variational inequalities", J. Math. Anal. Appl. 196 (1996) 314-331.

[26] C. T. Kelley, Iterative methods for linear and nonlinear equations (SIAM, Philadelphia, 1995).

[27] S. McCormick, "Multigrid methods for variational problems: general theory for the V-cycle", SIAM J. Numer. Anal. 22 (1985) 634-643.

[28] L. Qi and J. Sun, "A nonsmooth version of Newton's method", Math. Program. 58 (1993) 353-368. 
[29] W. Queck, "The convergence factor of preconditioned algorithms of the Arrow-Hurwicz type", SIAM J. Numer. Anal. 26 (1989) 1016-1030.

[30] T. Rusten and R. Winther, "A preconditioned iterative method for saddlepoint problems", SIAM J. Matrix Anal. Appl. 13 (1992) 887-904.

[31] Y. Saad and M. H. Schultz, "GMRES: a generalized minimal residual algorithm for solving nonsymmetric linear systems", SIAM J. Sci. Comput. 7 (1986) 856-869.

[32] D. J. Silvester and A. J. Wathen, "Fast iterative solution of stabilized Stokes systems part II: using general block preconditioners", SIAM J. Numer. Anal. 31 (1994) 1352-1367.

[33] P. Tseng, "Applications of a splitting algorithm to decomposition in convex programming and variational inequalities", SIAM J. Control Optim. 29 (1991) 119-138.

[34] A. J. Wathen and D. J. Silvester, "Fast iterative solution of stabilized Stokes systems part I: using simple diagonal preconditioners", SIAM J. Numer. Anal. 30 (1993) 630-649. 